Revue d'histoire du XIXe siècle

Société d'histoire de la révolution de 1848 et des

révolutions du XIXe siècle

Religion, politique et culture au XIXe siècle

\title{
John Plunkett, Queen Victoria, First Media Monarch, Oxford, Oxford University Press, 2003, 256 p.
}

\section{Philippe Chassaigne}

\section{OpenEdition}

Journals

Édition électronique

URL : http://journals.openedition.org/rh19/634

DOI : 10.4000/rh19.634

ISSN : $1777-5329$

Éditeur

La Société de 1848

Édition imprimée

Date de publication : 1 juin 2004

Pagination : 184-187

ISSN : 1265-1354

Référence électronique

Philippe Chassaigne, « John Plunkett, Queen Victoria, First Media Monarch, Oxford, Oxford University Press, 2003, 256 p. ", Revue d'histoire du XIXe siècle [En ligne], 28 | 2004, mis en ligne le 19 juin 2005, consulté le 22 septembre 2020. URL : http://journals.openedition.org/rh19/634 ; DOI : https://doi.org/ $10.4000 /$ rh19.634

Ce document a été généré automatiquement le 22 septembre 2020

Tous droits réservés 


\title{
John Plunkett, Queen Victoria, First Media Monarch, Oxford, Oxford University Press, 2003, 256 p.
}

\author{
Philippe Chassaigne
}

1 Cet ouvrage, tiré de la thèse de doctorat soutenue par John Plunkett, Junior Research Fellow (allocataire de recherche) en anglais à l'université d'Exeter, aborde la question de la mise en image(s) de la monarchie britannique, partant du principe que les media sont «l'un des principaux moyens par lesquels [elle] maintient sa traditionnelle prééminence » (p. 1).

2 L'un des éléments très positifs de son livre est d'étudier un segment chronologique souvent délaissé, à savoir, la première partie du règne de Victoria, de 1837 à $1870^{1}$. De fait, cette période est marquée par des difficultés et des contestations : en 1837, à l'avènement de la reine, le prestige de la monarchie britannique était au plus bas, ses prédécesseurs George IV (1820-1830) et Guillaume IV (1830-1837) n'ayant pas eu l'art et la manière de se faire apprécier de leurs sujets. Les premières années du règne de Victoria virent les parlementaires renâcler pour voter sa liste civile puis celle de son mari, Albert de Saxe-Cobourg, qu'elle épousa en 1840 - ce mariage avec un prince allemand ne fut d'ailleurs pas sans susciter de multiples réactions teintées de xénophobie. En outre, la période 1837-1848 correspond à la vague du chartisme, ultime avatar d'un radicalisme qui aurait véritablement pu évoluer en un mouvement révolutionnaire, et les chartistes ne se privèrent pas de dénoncer l'institution monarchique. Les années 1850 furent moins agitées, mais le décès du prince Albert en 1861 ouvrit une nouvelle "crise de confiance» envers la souveraine: accablée de chagrin, vivant littéralement en recluse, elle négligea les devoirs de sa charge, se coupant de ses sujets et permettant au mouvement républicain de connaitre son apogée historique. Comme on le sait, la maladie du prince de Galles, en novembre 1871, qui faillit lui être fatale, et sa guérison inespérée, provoquèrent le retournement de l'opinion et la nouvelle décennie marqua le début de "l'histoire d'amour» des Britanniques avec leur monarchie. La période 1837-1870 coïncide par ailleurs en 
Grande-Bretagne avec l'explosion des moyens de communication de masse, avec une production d'imprimés et d'images de plus en plus diversifiée et touchant des publics toujours plus nombreux. Ceci ne pouvait pas manquer d'influer sur les représentations du souverain, leur donnant, à la fin de la période, une omniprésence qui contrastait avec - et la compensait - la perte de substance politique que la fonction monarchique avait connu dans le même temps.

C'est l'un des thèmes clefs du livre, mais cette grille d'analyse n'est pas totalement nouvelle: David Cannadine en avait déjà développé certains aspects dans sa contribution à l'ouvrage bien connu d'Éric Hobsbawm et de Terence Ranger, The Invention of Tradition ${ }^{2}$. Toutefois, Plunkett l'applique à un objet plus étroitement circonscrit : l'imprimé à large circulation. Il identifie plus précisément cinq vecteurs de la diffusion de cette mise en images de la monarchie : les engagements protocolaires; les portraits royaux; les représentations graphiques, toujours plus nombreuses et souvent satiriques - au moins jusqu'à un certain point -, dans la presse populaire ; la photographie; le «reportage royal» (royal reporting), qui, à la fin de la période, s'affirme comme un genre journalistique à part entière. À chaque décennie, ou peu s'en faut, un medium nouveau vient se superposer aux vecteurs déjà existants, contribuant ainsi à la dissémination, au foisonnement, sinon à l'omniprésence, de l'image royale. En outre, J. Plunkett montre que le centre de l'attention s'est rapidement déplacé de la personne de la souveraine à son cercle familial immédiat (le prince Albert et leurs enfants) : l'exaltation de la "monarchie familiale " (family monarchy), généralement chronologiquement datée de la seconde moitié du règne de Victoria, est en fait perceptible dès les années 1840 .

Pour autant, cette image n'était initialement pas uniformément louangeuse : certes, la grande masse des publications exprimait clairement des sentiments pro-monarchiques et présentait une description complaisante, sinon carrément obséquieuse, des Royals ; mais l'intérêt de la période tient à l'existence d'un nombre non négligeable de publications infiniment plus critiques. Outre, bien sûr, les revues radicales, qui ne mettaient pas leur drapeau dans leur poche, il existait encore, au cours des premières décennies du règne de Victoria, tout un ensemble de publications satiriques (Figaro in London, Penny Satirist, Reynold's Newspaper, ou encore le célèbre Punch) qui prenaient peu de gants pour traiter de l'institution monarchique. La question du mariage royal (1839-1840), souvent ramenée à la quête quasi frénétique d'un prétendantreproducteur qui permette d'éviter que Victoria ne demeurât stérile, à l'instar de la reine Élisabeth ${ }^{\text {ere }}$, permit à ces organes de presse de décocher des piques savoureuses. Ce ton critique tend toutefois à disparaitre au fur et à mesure que l'on avance dans le règne de la souveraine, sans doute en raison de l'avènement de nouveaux vecteurs de diffusion qui, par leur caractère justement innovant, attirent l'attention du public: l'essor de la photographie est, de ce point de vue, particulièrement suggestif, permettant aux images édifiantes qu'elle diffusa massivement de reléguer les gravures satiriques aux oubliettes.

Toutefois, l'auteur est dès l'abord conscient de la complexité de la relation entre ces deux éléments, qui s'instrumentalisent réciproquement. Il démontre ainsi le savant jeu d'adaptation par lequel les membres de la famille royale surent, en dépit, souvent, de réelles réticences en leur for intérieur, se rendre accessibles aux media, intégrer leurs attentes pour projeter auprès de leurs sujets l'image qu'ils souhaitaient donner. De ce point de vue, la "guerre des Galles» des années 1990, entre le prince Charles et la 
princesse Diana, perd beaucoup de son caractère apparemment novateur. De fait, la première partie du règne de Victoria apparaît bien comme le moule dans lequel s'est coulée la politique de communication de la monarchie pour le siècle et demi qui devait suivre.

Le corollaire de la thèse de Plunkett est que cette visibilité permit à la monarchie de survivre en s'auto-justifiant, la fonction médiatique venant, en quelque sorte, prendre la relève d'une fonction politique que le développement du parlementarisme et du gouvernement de Cabinet rendait obsolète. Bien que devenue «inutile», les Britanniques, ou du moins leur majorité, ne songeaient pas à en demander l'abolition, dans la mesure où un spectacle médiatique soigneusement orchestré démontrait qu'elle avait toujours une utilité. Cette thèse d'une "ré-ivention" permanente de la monarchie pour sauvegarder son existence n'est pas, encore une fois, totalement nouvelle: ainsi, pour Frank Prochaska, c'est le développement de la fonction caritative-et, plus encore, son exploitation médiatique - de la monarchie britannique qui lui permit de maintenir son existence ${ }^{3}$. John Plunkett apporte donc des éléments factuels nouveaux à un processus par ailleurs assez bien documenté, et c'est un point tout à fait important. Son étude est en revanche moins convaincante - mais, pouvait-il en être autrement, dans la mesure où on se situe alors à la marge de son sujet initial - lorsqu'il traite, en quelques pages de conclusion et de façon très cavalière, de la période postérieure (fin du XIX et $\mathrm{XX}^{\mathrm{e}}$ siècles). Son analyse sur le « déclin, lent mais sûr et irrésistible » de la monarchie (p. 245) fait bon marché des sentiments populaires qui se manifestent de façon récurrente, lorsqu'il ne tombe pas, purement et simplement, dans des interprétations tendancieuses (par exemple, sur le manque d'écho populaire que le Jubilé d'Or de 2002 est censé avoir rencontré). Il ne semble pas voir la façon dont les compte-rendus des heurs et malheurs des Royals dans la presse populaire ont pu se substituer aux célébrations officielles auto-instituées. Tout, plutôt que l'indifférence? La recette semble bien avoir été comprise, il y a plus de 150 ans, par Victoria elle-même.

\section{NOTES}

1.. Cette période correspond d'ailleurs à celle qui est couverte dans l'ouvrage de Monica CHARLOT, Victoria. Le pouvoir partagé, Paris, Éditions Flammarion, 1989, qui pourra servir de contrepoint utile.

2.. David CANNADINE, «The Context, Performance and meaning of Ritual : the British Monarchy and the "Invention of Tradition", c. 1820-1977 », dans Eric HOBSBAWM et Terence RANGER (ed.), The Invention of Tradition, Cambridge, Cambridge University Press, 1983.

3.. Frank PROCHASKA, Royal Bounty. The Making of a Welfare Monarchy, New HavenLondres, Yale University Press, 1995. 